\title{
Matching Demand with Supply in the Smart Grid using Agent-Based Multiunit Auction
}

\author{
Tri Kurniawan Wijaya \\ École Polytechnique Fédérale de Lausanne \\ Switzerland \\ Email: tri-kurniawan.wijaya@epfl.ch
}

\author{
Kate Larson \\ University of Waterloo \\ Canada \\ Email: klarson@uwaterloo.ca
}

\author{
Karl Aberer \\ École Polytechnique Fédérale de Lausanne \\ Switzerland \\ Email: karl.aberer@epfl.ch
}

\begin{abstract}
Recent work has suggested reducing electricity generation cost by cutting the peak to average ratio (PAR) without reducing the total amount of the loads. However, most of these proposals rely on consumer's willingness to act. In this paper, we propose an approach to cut PAR explicitly from the supply side. The resulting cut loads are then distributed among consumers by the means of a multiunit auction which is done by an intelligent agent on behalf of the consumer. This approach is also in line with the future vision of the smart grid to have the demand side matched with the supply side. Experiments suggest that our approach reduces overall system cost and gives benefit to both consumers and the energy provider.
\end{abstract}

\section{INTRODUCTION}

Currently, electricity markets are designed so that the electricity supply has to fulfill the demand. When the demand increases rapidly, several problems occur, such as the possibility of power failures and high generation costs as expensive generators are turned on to fulfill demand for short peak periods. One vision for the smart grid has been to reverse this, and instead have demand match the available supply [10]. In this paper we propose a method towards achieving this end.

The key to our method is the explicit cut of the peak to average ratio (PAR) of the electricity load generated. We introduce an approach which cuts PAR and then lets consumers adapt by using an auction for redistributing the load. As others have proposed previously, automated intelligent agents can be used to represent consumers in these auctions, making the entire process seamless from the consumers' perspective [9], [11], [13].

Both the challenges of obtaining low PAR and the advantages have been widely investigated [1], [3], [12]. However, to the best of our knowledge, no other work proposes to explicitly cut PAR and let the demand side adapt to the supply. Many other approaches which have investigated cutting PAR have focussed on having the demand side voluntarily adjust their consumption (given various incentives) in order to reduce peak load [7], [8], [5].

Using auctions in electricity markets has been both proposed in the literature and been used in practice, see for example [2], [4]. In our work, however, we take a close look at the relationship between produces and consumers and realize the vision of matching demand with supply while also considering 978-1-4673-5494-3/13/\$31.00 (c) 2013 IEEE demand satisfiability and minimum load guarantees, while also supporting the possibility of additional benefits for each side. The work most similar to that proposed here looked at the use of service curves [6]. This work is similar in that it also imposed certain restrictions on the consumers' consumption. However, in their work, the producers and consumers had to agree in advance on the service curves contract, reducing the ability to adapt to changing supply conditions.

This paper makes the following contributions:

- We propose an algorithm to explicitly cut PAR and prove its soundness and completeness.

- We introduce a (multiunit) auction for distributing load after the PAR cut. We provide a minimum load guarantee for all consumers, and show that the auction support truthful myopic bidding.

- Our simulations illustrate that our method benefits both consumers and producers.

The rest of the paper is organized as follows. We describe our model and other basic notions in Section II. In Section III we describe the algorithm for cutting PAR and prove that it is both sound and complete, while in Section IV we present our auction for distributing available load. Our experimental results are presented in Section V, which conclusions and further discussions in Section VI.

\section{Preliminaries}

\section{A. Load Modeling}

Load. Let $\mathcal{N}$ be a set of consumers and $\mathcal{T}=\left\{t_{1}, \ldots, t_{|\mathcal{T}|}\right\}$ be a set of uniform time slots in a day. We denote the electricity load (or simply load) needed by consumer $i \in \mathcal{N}$ in time slot $t \in \mathcal{T}$ as $L_{i}(t)$. Hence, the total load of consumer $i$ for a day is given by:

$$
L_{i}=\sum_{t \in \mathcal{T}} L_{i}(t)
$$

In addition, we define total load per time slot over all consumers as:

$$
L(t)=\sum_{i \in \mathcal{N}} L_{i}(t)
$$

PAR. Peak to average ratio $(P A R)$ is a commonly used measurement to express how the peak compares to the average 
load:

$$
\operatorname{PAR}(L)=\frac{|\mathcal{T}|}{\sum_{t \in \mathcal{T}} L(t)} \max _{t \in \mathcal{T}} L(t)
$$

\section{B. Multiunit Auction}

Before introducing our auction (in Section IV), in this section we briefly explain the basics of multiunit auctions.

Auction. We use a uniform price auction, where each winning bidder pays the same price for each item/resource they win. The price paid is the price of the highest non-winning bidder. Given $R$ resources and $\mathcal{N}$ as a set of agents or bidders, we denote a bid of an agent $i \in \mathcal{N}$ as a 2-tuple $\left(r_{i}, v_{i}\right)$ where $r_{i}$ is the number of resources desired by agent $i$ and $v_{i}$ is her valuation (the price $i$ willing to pay for each resource she wins).

Winners. The resources are won by the $k$ highest bidders. Let $W \subseteq \mathcal{N}$ be the set of $k$ agents who win the auction, then we require that:

(i) the winners are the highest bidders: $\forall i, j\left(v_{i} \geq v_{j}\right)$ where $i \in W$ and $j \in \mathcal{N} \backslash W$,

(ii) we allocate, tentatively, the maximum number of resources: if $W \subset \mathcal{N}$ then $\left(\sum_{i \in W} r_{i}\right) \geq R$, and

(iii) $W$ is the smallest set of winners: $\forall W^{\prime}\left(\sum_{i \in W^{\prime}} r_{i}\right)<R$ where $W^{\prime} \subset \mathcal{N}$.

Price. The price paid by each winner $i \in W$ is the valuation of the highest non-winning bidder, i.e., $p^{*}=v_{j}$ where $\forall j^{\prime}\left(v_{j} \geq\right.$ $\left.v_{j}^{\prime}\right)$ where $j, j^{\prime} \in \mathcal{N} \backslash W$. Note that when $W=\mathcal{N}$ then there are two possibilities, either all bidders pay 0 or pay the reserve price (a price which is fixed by the auctioneer as the minimum price for a resource).

Resource distribution. We sort the winning bidders by their valuation in ascending order. The resources are distributed to the winners starting from the highest bidders. Hence, there could be the case where the lowest winning bidder gets resources less than what she desires. In this case, she has an option to walk away (cancel her participation in the auction) or accept the resources offered.

Example 1 (Multiunit Auction): A company would like to sell 6 resources. Then,

- bidder 1 would like to buy 2 resources at $\$ 12$,

- bidder 2 would like to buy 3 resources at $\$ 10$,

- bidder 3 would like to buy 3 resources at $\$ 8$, and

- bidder 4 would like to buy 1 resources at $\$ 6$.

- bidder 5 would like to buy 2 resources at $\$ 5$.

Hence the winners of the auction are bidder 1, 2, and 3 . Bidder 1, 2, and 3 got 2, 3, and 1 item respectively where each of them have to pay $\$ 6$ for an item. In this case, since the total demand of bidder 3 is not met, she can decide whether to take the 1 item offered and pay $\$ 6$, or withdraw from the auction (pay nothing and receive no item).

\section{PAR-CUT}

We cut $P A R(L)$ by a cut percentage, $c$, resulting in a new load vector $L^{\prime}$ :

$$
(1-c) \cdot \operatorname{PAR}(L)=\operatorname{PAR}\left(L^{\prime}\right),
$$

and

$$
\sum_{t \in \mathcal{T}} L(t)=\sum_{t \in \mathcal{T}} L^{\prime}(t) .
$$

When condition in Eq. 3 is met, using Eq. 1 we can rewrite Eq. 2 as:

$$
(1-c) \cdot \max _{t \in \mathcal{T}} L(t)=\max _{t \in \mathcal{T}} L^{\prime}(t) .
$$

In Algorithm 1 we provide a technique to explicitly cut the $P A R$ of the original load generated by consumer demand. The returned result is a load whose $P A R$ has been cut. Cutting $P A R$ while maintaining overall amount of load (as described in Section (III) means that there are some amount of load shifted from their original time slot. In this algorithm we aim to minimize the shift distance by first attempting to shift to a neighboring time slot.

There are several helper methods used in Algorithm 1 .

- findPeak $(L)$ : returns the peak load of a load vector $L$.

- $\min (A, B)$ : returns the smallest between two number $A$ and $B$.

- $\operatorname{shift}\left(x, L, t_{1}, t_{2}\right)$ : moves $x$ amount of load from $L_{t_{1}}$ to $L_{t_{2}}$.

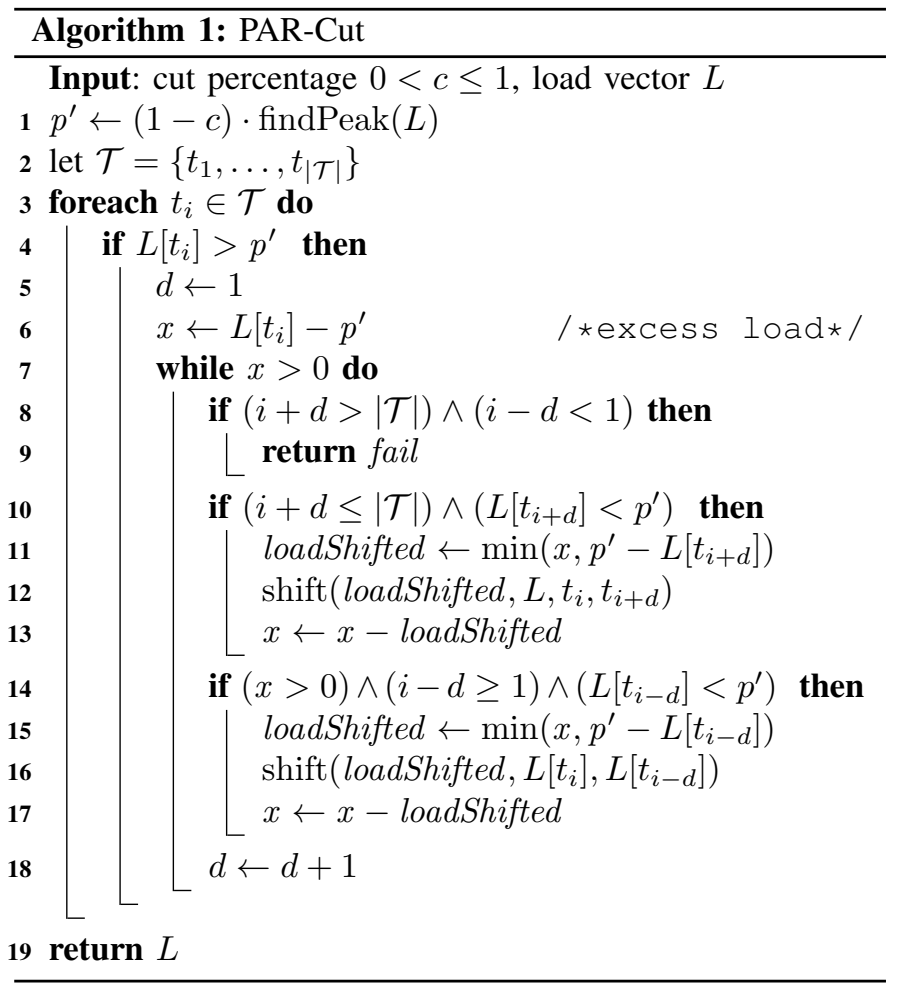

Algorithm 11 receives input $c$ as the cut percentage and $L$ as the original load whose $P A R$ is to be cut. We use Eq. 4 to define the new target peak $p^{\prime}$ (line 11). Then, for each time slot $t_{i}$, we verify whether the load at $t_{i}$ exceeds $p^{\prime}$ (line 4). If this is the case, then we try to shift it (line 5 18). We first try to shift the load at $t_{i}$ to its neighboring time slot by 
incrementing variable $d$ for checking time slot $t_{i+d}$ (line 10) and $t_{i-d}$ (line 14). However, when we have investigated all time slots and there is still an amount of load to be shifted, then we conclude that it is not possible to do the cut, and the algorithm returns with failure (line 9).

Example 2 (PAR-cut): Let us assume that we have a set of time slots $\mathcal{T}=\left\{t_{1}, t_{2}, \ldots, t_{24}\right\}$, and load $L_{t_{19}}=5 \mathrm{kWh}$, $L_{t_{18}}=L_{t_{20}}=2 \mathrm{kWh}$ and $L_{t_{i}}=1 \mathrm{kWh}$, where $1 \leq i \leq 17$, and $21 \leq i \leq 24$. In this case we have $P A R=4$. Furthermore, let us assume that we want to cut the $P A R$ by $40 \%$ cut percentage. Hence, our new peak $p^{\prime}=3$ (see line 1 ). First, Algorithm 1 detects that we have an excess load at time slot $t_{19}$, and the excess load $x=2 k W h$. Then, it proceeds with distributing the load to the neighboring time slot, $t_{20}$ and $t_{18}$. This makes $L_{t_{18}}=L_{t_{19}}=L_{t_{20}}=3 k W h$. At the end of this step, the excess load $x=0$, and the algorithm returns the newly modified load vector.

For the proof of the soundness and the completeness of the algorithm, we refer to the input load to the algorithm as $L$, and the resulting load as $L^{\prime}$.

Soundness: We need to show that if the algorithm returns $L^{\prime}$, then this is correct (having property shown in Eq. 3 and 4). Satisfying condition in Eq. 3 is straightforward since we never decrease or add new load. The only modification we apply is shifting the load from one time slot to another. Next, we always make sure that the maximum load in a time slot never exceeds $p^{\prime}$ (see line 4). This makes the condition in Eq. 4 hold.

Completeness: We need to show that if the algorithm returns fail, then it is not possible to cut the load (as specified by the cut percentage $c$ ). The only condition where the algorithm returns fail is when it reaches line 9. This means that we still have an excess load, and we have no slot left with load less than $p^{\prime}$. Hence, in order to satisfy the condition in Eq. 3, we have to put this excess load in some time slot. But this will make this time slot have load greater than $p^{\prime}$ which is not permitted by Eq. 4

\section{Multiunit Auction for LoAd Distribution}

In this section we explain how we run multiunit auction to distribute the cut load (the resulting load from Algorithm 1). The auction is run either in the beginning of the day or a day before. It is held in several rounds until all loads are distributed. We use $\mathcal{N}$ as the set of consumers and refer to agent $i$ as the bidding agent representing consumer $i \in \mathcal{N}$.

\section{A. Initial condition}

Let $L^{\prime}$ denote the cut load vector on which we are going to run the auction, and let $L$ denote the original load vector. For a time slot $t \in \mathcal{T}$, whenever $L^{\prime}(t) \geq L(t)$, the demanded loads are distributed to the consumers.

Let $L_{i}^{x}$ be the actual load vector for consumer $i \in \mathcal{N}$ (the actual loads delivered to consumer $i$ 's residence) obtained in round $x$ of the auction and let round 0 be the initial step before the auction begins. Then, we have consumer $i$ 's actual load: $L_{i}^{0}(t)=L_{i}(t)$ for $L^{\prime}(t) \geq L(t)$ and time slot $t \in \mathcal{T}$.

\section{B. Minimal Load Guarantee}

It could be the case that at time slot $t$ there is a consumer $i \in \mathcal{N}$ who does not get any load because all loads have been won by other consumers. It means that at time $t$ consumer $i$ does not have any electricity. This could happen when $L^{\prime}(t)<$ $L(t)$ for some $t \in \mathcal{T}$. That is, when we face a short of supply condition (because of the cut) where the load supply at time $t$ is less than the actual consumers' demand.

In order to prevent this condition from happening, we set $m$ as the minimal amount of load guaranteed for each consumer to have when we face a short of supply, i.e. for some time slot $t \in \mathcal{T}$, when $L^{\prime}(t)<L(t)$ :

(i) supply guarantee: $L^{\prime}(t) \geq m \cdot|\mathcal{N}|$,

(ii) minimal load guarantee: $L_{i}^{0}(t)=m$ for all $i \in \mathcal{N}$.

\section{Initial Pricing}

To protect the energy company getting paid 0 when we run the auction (this can happen when $W=\mathcal{N}$ ), we define the reserve price per Wh for each time slot $t \in \mathcal{T}$ as

$$
p(t)=\frac{\cos t(L(t))}{L(t)} .
$$

We define $\cos t(L(t))=c_{1} L(t)^{2}+c_{2} L(t)+c_{3}$ (increasing and convex), such that time slots having higher load is more expensive [8].

\section{The Auction: Matching Demand Against Supply}

We use the multiunit auction as described in Section II-B with some modifications:

1) An agent submits a set of bid $\left\{b_{1}, \ldots, b_{|\mathcal{T}|}\right\}$ all at once, where $b_{i}$ is a bid for the load on time slot $t_{i} \in \mathcal{T}$.

2) Since it could be the case that not all loads are distributed to consumers on one round of the auction, we run multiple rounds of the auction where the loads available for the next round are the loads left from the current round.

3) Bids (how much load an agent want and at what price) are placed simultaneously but separately for each time slot.

4) The winners are determined for each time slot.

5) The auction is terminated when there is no load left or there is no bid submitted.

Initial condition. Let $L^{x}(t)$ be the loads left (after it has been distributed to the winners) after the $x^{t h}$ round of the auction. Thus, we denote the initial loads available before the auction begins as $L^{0}(t)=L^{\prime}(t)-\sum_{i \in \mathcal{N}} L_{i}^{0}(t)$ for time slot $t \in \mathcal{T}$, where round 0 is the initial step before the auction begins and $L^{\prime}$ is the cut load vector.

Bid. In each round $x$ of the auction, agents place a bid on $L^{x-1}(t)$ using their own valuation to get some load they desired for each time slot $t \in \mathcal{T}$. However, if the load available in $L^{x-1}(t)$ is less than what an agent needs, then she will place a bid on $L^{x-1}\left(t^{\prime}\right)$ where $t^{\prime}$ is the closest time slot to 
$t$ such that $L^{x-1}\left(t^{\prime}\right)$ is greater than what she needs. This is often called as myopic best response strategy, i.e. each agent is interested to maximize her own utility given the current condition. Note that it is possible that a sophisticated agent might be able to manipulate the system by reasoning and acting over several time steps and do a bit better. However, this involves sophisticated reasoning and could well be too complex for more simple bidders. And we will show that the optimal myopic strategy has attractive properties.

Winners determination. The winners are determined separately for each time slot, i.e. we have a set of winners $W^{x}(t)$ for each time slot. Next, the resources available are updated to $L^{x}(t)$ by considering the load that has been distributed to the winners.

Demand satisfiability. Let $x^{*}$ be the final round of the auction. In addition, for a consumer $i \in \mathcal{N}$ let $L_{i}^{\prime}(t)=$ $\sum_{x=0}^{x^{*}} L_{i}^{x}(t)$ be the sum of load she obtained through the auction for each time slot $t \in \mathcal{T}$. Then, we require for consumer :

$$
\left(\sum_{t \in \mathcal{T}} L_{i}^{\prime}(t)\right)=\left(\sum_{t \in \mathcal{T}} L_{i}(t)\right)
$$

That is, the total load obtained by a consumer should be the same as her total electricity demand.

Truthful bidding is a myopic best response. We prove that the auction supports truthful bidding, i.e. there is no incentive for agents to lie about their valuation.

Proof: Assume that there is still load that needs to be acquired by agent $i$ on round $x$. Then, there are two cases:

1) $L^{x-1}(t)$ is equal or larger than what agent $i$ needs. Then, agent $i$ will bid on it. Assume that $v_{i}(t)$ is the agent $i$ valuation for the load in time slot $t$. There are two cases:

a) Agent $i$ does not win. If agent $i$ bid an amount $<v_{i}(t)$, it could not make her win. However, if she bid an amount $>v_{i}(t)$, she could win. But then she has to pay $v^{\prime}$ (the bid of the highest non-winning bidder), and it is also possible that $v^{\prime}>v_{i}(t)$. In this case, agent $i$ loss (because she has to pay higher than her valuation of the electricity at that time).

b) Agent $i$ win. Assume that the highest non-winning bid is $v^{\prime}$. This is also the price that the winners have to pay. If agent $i$ bid $b^{\prime}$, and $v^{\prime} \leq b<v_{i}(t)$, then she still has to pay $v^{\prime}$ (she does not gain any additional benefit). However, if agent $i$ bid $<v^{\prime}$, then she lost. Hence, she does not get the load she want. And if agent $i$ bid $>v_{i}(t)$, this also does not give any additional benefit because she still has to pay $v^{\prime}$.

2) $L^{x-1}(t)$ is less than what agent $i$ need. Then, agent $i$ choose the closest time slot to $t$, that is $t^{\prime}$, such that $L^{x-1}\left(t^{\prime}\right)$ is equal or larger that what agent $i$ need. Then, the same the argument as in the two cases above $1 \mathrm{a}$ and $1 \mathrm{~b}$ ), are applied here by replacing $t$ with $t^{\prime}$.

\section{E. Consumer's Load Shifting}

Note that a bid for an amount of load at time slot $t \in \mathcal{T}$ placed by consumer $i$ does not necessarily comes from her actual need, from $L_{i}(t)$. Consider a simple example when consumer $i$ actually still needs to obtain her load for time slot $t^{\prime} \in \mathcal{T}$ in the current round $x$, but $L^{x}\left(t^{\prime}\right)=0$. Since there is no more load available in time slot $t^{\prime}$, then she has to place a bid for a different time slot $t^{\prime \prime} \in \mathcal{T}$. When she wins the bid, load shifting for consumer $i$ occurs, i.e. she shifts an amount of electricity consumption from time $t^{\prime}$ to $t^{\prime \prime}$.

\section{F. Consumer's Utility}

We define consumer's utility after the auction by two terms:

(i) cost: we compute the cost paid by a consumer at the end of an auction.

(ii) shift percentage: in order to measure a consumer's inconvenience, we calculate the percentage of load that she has to shift. The shift percentage of consumer $i$ is:

$$
\frac{\sum_{t \in \mathcal{T}}\left|L_{i}^{\prime}(t)-L_{i}(t)\right|}{2 \cdot \sum_{t \in \mathcal{T}} L_{i}(t)} .
$$

\section{EXPERIMENTS}

In the experiments, we show the effects of the auction to consumer's utilities for different type of consumers. In addition, we investigate whether there is any additional benefit for the consumer and/or the energy provider from running this auction.

\section{A. Experiment Setup}

In our experiments, we use an hourly time slot, i.e. $\mathcal{T}=\{1, \ldots, 24\}$, and total consumers, $|\mathcal{N}|=10000$. Then, we provide appliance usage setting to simulate consumers' electricity demand. For the cost, any increasing and convex function can be used. In particular, we set $\operatorname{cost}(L(t))=$ $\left(\left(L(t)+q_{1}\right) /\left(q_{2} \sqrt{|\mathcal{N}|}\right)\right)^{2}$ in order to include the energy company's marginal benefit, $q_{1}$, and to control the price's growth, $q_{2}$ (we set $q_{1}=100$ and $q_{2}=1000$ ).

For consumer valuations (see Section II-B, we use $\alpha \cdot p(t)$, where $p(t)$ is the reserve price at time slot $t \in \mathcal{T}$ (see Section IV-C). In order to set $\alpha$ among consumers, we use two types of distributions

1) US distribution: for each consumer, her $\alpha$ is drawn from a set $\{1.0,1.3,1.5,1.6,1.9\}$ with distribution $0.4,0.2,0.2$, 0.1 , and 0.1 respectively. This distribution is inspired by the US households wealth distribution in 20041

2) Uniform distribution: for each consumer, her $\alpha$ is drawn uniformly from set $\{1.0,1.1,1.2 \ldots, 1.9\}$.

Each experiment is run 10 times and all results presented are with $95 \%$ confidence interval. However, the intervals almost cannot be seen because the deviations are very small.

${ }^{1}$ http://www.faculty.fairfield.edu/faculty/hodgson/courses/so11/stratification /income\&wealth.htm (accessed: 15 September 2012) 


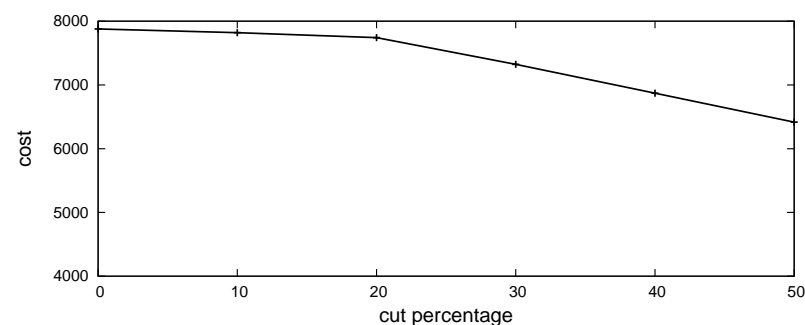

Fig. 1. Overall system cost decrease as the cut percentage getting larger. Simulation using 10000 households loads.

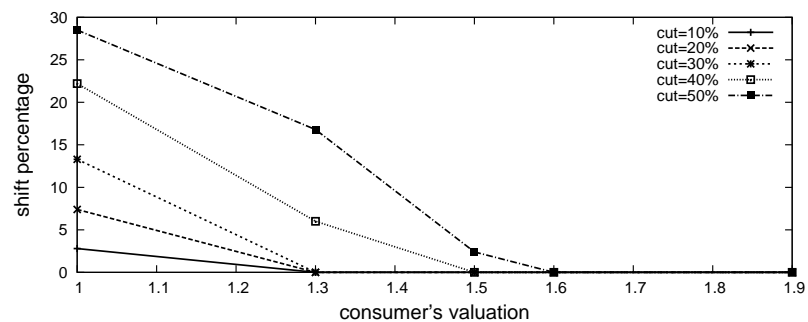

Fig. 2. Consumers' utility based on shift percentage measurement (defined in Section IV-F grouped by their valuation (US distribution).

\section{B. System Cost}

We present how the $P A R$ cut affects the system cost. We show the result up to $50 \%$ cut percentage, since in our setting, it is not possible to cut the PAR with $60 \%$ or more. As expected, the more we cut the PAR, the lower the overall system cost is. Our experimental results in Figure 1 shows that cutting the PAR up to $20 \%$ does not give significant advantage. However, cutting the PAR with $50 \%$ cut percentage give almost $20 \%$ overall cost reduction. It looks very promising, but one have to be more careful and look on how consumer's utility changes with increasing cut percentage (as we will show later in this section).

\section{Consumers' Utility}

We measure consumers' utility from two perspectives. First, we look at how much they need to shift their consumption. Second, we look at the total cost that they need to pay.

Shift percentage. The results from both distributions (US and uniform) depicted in Figure 2 and 3 shows the same trends, i.e. consumers with lower valuation are the ones who shift the most. This is expected since most of their demands which compete with higher valuation consumers are not satisfied (because they lost in the auction). Hence they need to shift their consumption (bid for another time slot). For consumers with valuation more than 1.7 we see no difference in their utility, and they do not shift at all. With this high valuation, we expect that they always win the auction and so see no difference in their supply.

Cost paid. Figure 2 and 3 both show the same trends that the higher the consumer valuation, the higher the cost. This phenomenon is both caused by the reserve price and the auction itself. Most of the consumers firstly will bid to satisfy their original consumption schedule, which happen most of the time in the peak time slot. Although we have already cut the

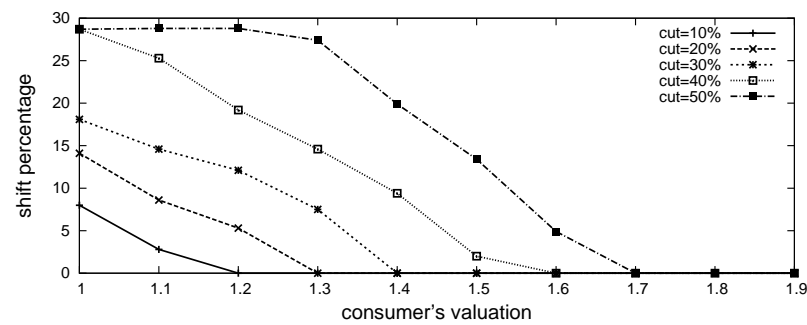

Fig. 3. Consumers' utility based on shift percentage measurement (defined in Section IV-F grouped by their valuation (uniform distribution).

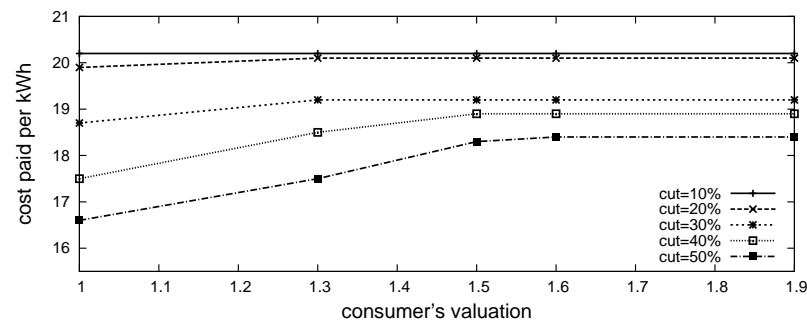

Fig. 4. Consumers' utility based on the total cost paid (defined in Section IV-F] grouped by their valuation (US distribution).

peak, the price of this time slot is still the highest compared to the other (this is the time slot with the highest load). In addition, since most of the consumers bid on this time slot, most likely the winners for this time slot will be the higher valuation consumers, and the highest non-winning bid (which will determine the price they have to pay) will also be high.

As expected, low valuation consumers pay lower costs. This can also be seen as the tradeoff (from the previous results) that they need to shift more than the consumers with high valuation. The setting with $50 \%$ cut percentage offer lower cost paid compared to the others, but it also caused the low valuation consumers to shift more (see Figure 2 and 3 .

\section{Consumers' Additional Benefit}

Compared to the current system, consumers gain an additional benefit using the PAR cut and the auction. Consumers can save up to almost $20 \%$ their electricity bill depending on the cut percentage implemented (see Figure 6). This happens without having to reduce the amount of their electricity consumption. For consumers with low valuation this saving can be seen as the tradeoff since they experience load shifting. However, what is more interesting is that this saving also applies to consumers with high valuations (in this case, higher than 1.6) who experience no load shifting at all (see Figure 2).

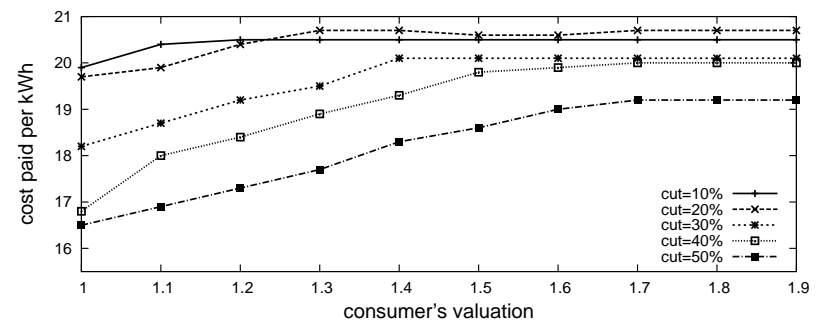

Fig. 5. Consumers' utility based on the total cost paid (defined in Section IV-F grouped by their valuation (uniform distribution). 


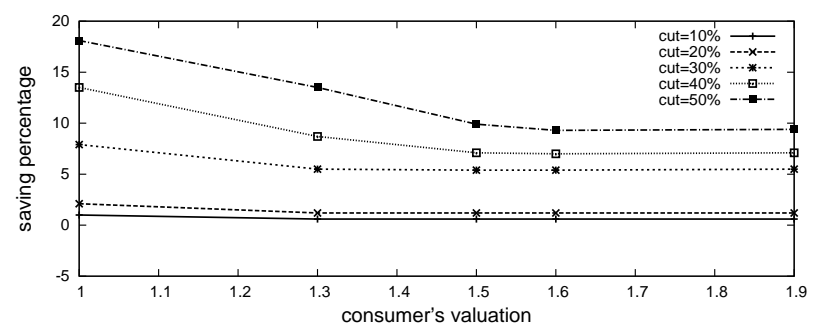

Fig. 6. Consumers' cost saving percentage using the auction and PAR cut compare to the current system. Valuation used: US distribution.

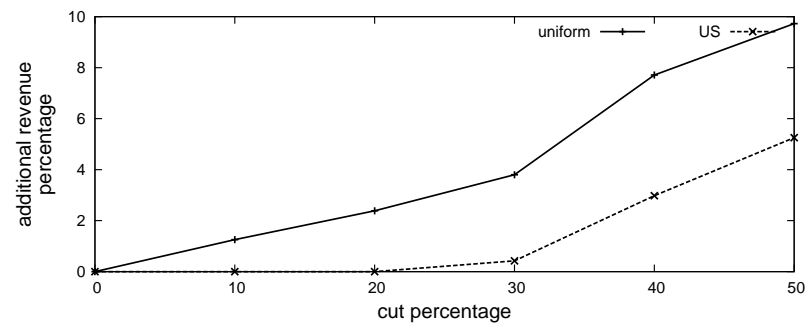

Fig. 7. Company addition revenue from the auction.

\section{E. Company's Additional Benefit}

In this experiment, we calculated the total cost paid by consumers and compared it to the energy company's cost model (the one that has to be paid by the consumers, as described in Section V-A. Figure 7 shows that the company experiences additional revenue by running the auction up to almost $10 \%$ depending on the PAR cut implemented. The larger PAR cut we have, the more peak loads are shifted. This will increase the price in the peak time slot because most likely the winners are consumers with high valuations, and the highest non-winning bid will most likely also be high.

\section{CONCLUSions AND Future Work}

In this paper, we addressed the future vision of the smart grid to match the demand with the supply. Instead of relaying the PAR cut to the consumer, we cut PAR explicitly while still maintaining the same load. Because the load available for each time slot are not necessarily the same as demanded by the consumers, we used a multiunit auction to distribute the load. Our experiments showed that the overall system cost is decreasing as we have larger $P A R$ cut percentages. We also showed that there is a tradeoff between the price per kWh paid by a consumer with the load shift percentage she experienced. In addition, the consumer with low valuation normally has bigger shift percentage and lower price per $\mathrm{kWh}$ than the consumer with high valuation. Another advantage is shown for the consumers that their total electricity cost is lower compare than their original electricity cost using the current setting (saving up to almost 20\%). While for the low valuation consumers this can be understood as the tradeoff for their load shifting, the saving also happens for high valuation consumers who does not have any load shifting. The energy company also reaped an additional benefit from running the auction by acquiring up to almost $10 \%$ additional revenue. This phenomenon made the proposed approach look promising.

This work can be extended by considering a case when a consumer considers non-myopic strategies and reasoning over multiple steps at once. Our conjecture is that weak consumers (low valuation) can benefit from bidding on nonpeak time slots for her needs in peak time slots. By bidding on non-peak time slots early, a weak consumer has a better chance to avoid competition with stronger consumers (who also have to shift to those time slots because of even stronger consumers). Furthermore, our approach can also be used for load distribution when we have load fluctuation (for example, because of the renewable energy sources). Because the essence of our approach is to match the demand with the supply, we can use it either when we have to cut the PAR, or when we have energy surplus to distribute.

\section{ACKNOWLEDGMENT}

The research leading to these results has received funding from the European Union's Seventh Framework Programme (FP7/2007-2013) under grant agreement no: 288322.

\section{REFERENCES}

[1] "Benefits of demand response in electricity markets and recommendations for achieving them," U.S. Dept. of Energy, Tech. Rep., 2006.

[2] J. Bower and D. Bunn, "Experimental analysis of the efficiency of uniform-price versus discriminatory auctions in the england and wales electricity market," Journal of Economic Dynamics and Control, vol. 25, no. 34, pp. $561-592,2001$.

[3] P. Cappers, C. Goldman, and D. Kathan, "Demand response in u.s. electricity markets: Empirical evidence," Energy, vol. 35, no. 4, 2010.

[4] J. Contreras, O. Candiles, J. De La Fuente, and T. Gomez, "Auction design in day-ahead electricity markets," IEEE Transactions on Power Systems, vol. 16, no. 1, pp. 88 -96, Feb 2001.

[5] T. Ganu, D. P. Seetharam, V. Arya, R. Kunnath, J. Hazra, S. A. Husain, L. C. De Silva, and S. Kalyanaraman, "nplug: a smart plug for alleviating peak loads," in Proceedings of the 3rd International Conference on Future Energy Systems: Where Energy, Computing and Communication Meet, ser. e-Energy '12. New York, NY, USA: ACM, 2012, pp. 30:1$30: 10$.

[6] J.-Y. Le Boudec and D.-C. Tomozei, "Demand response using service curves," in IEEE PES - ISGT, 2011.

[7] N. Li, L. Chen, and S. H. Low, "Optimal demand response based on utility maximization in power networks," in Proceedings of the 2011 IEEE Power \& Energy Society General Meeting, July 2011.

[8] A. Mohsenian-Rad, V. Wong, J. Jatskevich, R. Schober, and A. LeonGarcia, "Autonomous demand-side management based on gametheoretic energy consumption scheduling for the future smart grid," IEEE Transactions on Smart Grid, vol. 1, no. 3, pp. 320 -331, Dec. 2010.

[9] H. S. Oh and R. Thomas, "Demand-side bidding agents: Modeling and simulation," IEEE Transactions on Power Systems, vol. 23, no. 3, pp. 1050 -1056, Aug. 2008.

[10] S. D. Ramchurn, P. Vytelingum, A. Rogers, and N. R. Jennings, "Putting the 'smarts' into the smart grid: a grand challenge for artificial intelligence," Commun. ACM, vol. 55, no. 4, pp. 86-97, Apr. 2012.

[11] S. D. Ramchurn, P. Vytelingum, A. Rogers, and N. Jennings, "Agentbased control for decentralised demand side management in the smart grid," in The 10th International Conference on Autonomous Agents and Multiagent Systems - Volume 1, ser. AAMAS '11. Richland, SC: International Foundation for Autonomous Agents and Multiagent Systems, 2011, pp. 5-12.

[12] G. Strbac, "Demand side management: Benefits and challenges," Energy Policy, vol. 36, no. 12, pp. 4419 - 4426, 2008.

[13] P. Vytelingum, T. Voice, S. D. Ramchurn, A. Rogers, and N. R. Jennings, "Theoretical and practical foundations of large-scale agent-based microstorage in the smart grid," J. Artif. Intell. Res. (JAIR), vol. 42, pp. 765$813,2011$. 